\title{
A!
}

This is an electronic reprint of the original article.

This reprint may differ from the original in pagination and typographic detail.

Korhonen, Ari; Vivitsou, Marianna

\section{Digital storytelling and group work}

\section{Published in:}

ITiCSE 2019 - Proceedings of the 2019 ACM Conference on Innovation and Technology in Computer Science Education

DOI:

$10.1145 / 3304221.3325528$

Published: 02/07/2019

Document Version

Peer reviewed version

Please cite the original version:

Korhonen, A., \& Vivitsou, M. (2019). Digital storytelling and group work: Integrating the narrative approach into a higher education computer science course. In ITiCSE 2019 - Proceedings of the 2019 ACM Conference on Innovation and Technology in Computer Science Education (pp. 140-146). (Annual Conference on Innovation and Technology in Computer Science Education, ITiCSE). ACM. https://doi.org/10.1145/3304221.3325528

This material is protected by copyright and other intellectual property rights, and duplication or sale of all or part of any of the repository collections is not permitted, except that material may be duplicated by you for your research use or educational purposes in electronic or print form. You must obtain permission for any other use. Electronic or print copies may not be offered, whether for sale or otherwise to anyone who is not an authorised user. 


\title{
Digital Storytelling and Group Work:
}

\author{
Integrating the Narrative Approach into a Higher Education Computer Science Course
}

\author{
Ari Korhonen \\ Aalto University \\ ari.korhonen@aalto.fi
}

\author{
Marianna Vivitsou \\ University of Helsinki \\ marianna.vivitsou@helsinki.fi
}

\begin{abstract}
This study discusses the integration of digital storytelling and the narrative approach into a University level Computer Science course. The pedagogical intervention took place on a project basis. The plan involved student work in groups for the production of digital stories in three phases, including an abstract, a manuscript and a final story. The overall instructional design included workshops and lectures, online tutorials, and group work. The students were assigned to explore the topic of recursion. Face-to-face meetings for the coordination of group work were emphasized during lectures, workshops and project instructions.

The study uses qualitative research methods and the findings indicate two main patterns of group work. The first pattern follows from loose coordination and division of tasks among group members at the initial stages of the project. This results in documentarylike and program-based video stories. The second pattern involves tighter collaboration with face-to-face meetings for common task completion, video recording and editing, and manuscript improvement. This mode of work results in short-film style stories where recursion is well-represented. In both patterns, however, the videos present external rather than internal examples of recursion. As a result, the digital stories represent what the code does instead of how it does it.
\end{abstract}

\section{CCS CONCEPTS}

- Social and professional topics $\rightarrow$ Computing literacy;

\section{KEYWORDS}

Digital Storytelling; Narrative; Group Work; Critical Thinking; Higher Education; Computer Science; Data Structures; Algorithms

\section{ACM Reference Format:}

Ari Korhonen and Marianna Vivitsou. 2019. Digital Storytelling and Group Work:: Integrating the Narrative Approach into a Higher Education Computer Science Course. In Innovation and Technology in Computer Science Education (ITiCSE '19), July 15-17, 2019, Aberdeen, Scotland Uk. ACM, New York, NY, USA, 7 pages. https://doi.org/10.1145/3304221.3325528

Permission to make digital or hard copies of all or part of this work for personal or classroom use is granted without fee provided that copies are not made or distributed for profit or commercial advantage and that copies bear this notice and the full citation on the first page. Copyrights for components of this work owned by others than ACM must be honored. Abstracting with credit is permitted. To copy otherwise, or republish, to post on servers or to redistribute to lists, requires prior specific permission and/or a fee. Request permissions from permissions@acm.org.

ITiCSE '19, fuly 15-17, 2019, Aberdeen, Scotland Uk

(C) 2019 Association for Computing Machinery.

ACM ISBN 978-1-4503-6301-3/19/07 ..\$15.00

https://doi.org/10.1145/3304221.3325528

\section{INTRODUCTION}

While curricula open up to make room for Computer Science (CS) nowadays, computer scientists and theorists (e.g. [2, 6, 12,26]) point out the need to include both social and technical aspects in the way we think about the field. As, for example, software-based technologies increasingly shape our social, political and economic lives, the need becomes more and more pressing to view CS elements, such as algorithms, not only technically, but also embedded culturally, contextually and ethically [12].

This perspective would require an interdisciplinary approach in the CS curricula. The integration of digital storytelling (DST) as a pedagogical method informed by the principles of narrative approach would fit well with the need for interdisciplinarity. The narrative approach focuses on the development of plot and character for telling stories with digital technologies that can be shared with peers and other interested audiences. Concerning implementation, this view raises questions about what methods and techniques would best fit with the principles of narrative DST in combination with a socio-technically grounded CS education.

Earlier studies on DST in schools [10, 15, 18, 19, 21, 23, 24, 27-30] have shown the potential of the method to open up spaces for student involvement in group work to tell and share video stories with peers online. As the process requires to deal with heterogeneous elements for the synthesis of stories with a beginning, a middle and an end [11,22], the students enter a process of resignification and, thus, develop a deeper understanding of the object of study in a contextualized way [27, 29]. In addition, previous research has shown that the process allows storytellers to structure their personal narratives in time $[8,32]$ through the accounts of events and the building of characters that DST entails. Based on these considerations, we assumed that the DST pedagogy would open up the opportunity for students with CS as part of their major and minor studies to work together, set shared purposes and use digital technologies to tell stories based on CS concepts and content. As CS concepts are abstract, our hypothesis was that the task to build stories and characters for coherent and clear representations would lead students to more concrete configurations. Thus, the students were asked to look for manifestations of recursion in real life and in nature. In this way, the approach to recursion would be contextualized and, thus, more practical than theoretical.

Although there have been studies on DST in Higher Education [9], to our knowledge, its pedagogical integration in CS courses has not been studied to the point of writing. In addition, in the case of [20]'s study with university students, storytelling is scripted and concerns the development of a specific software rather than the narrative structure. Our study, however, focuses on student's own work with story lines and characters authored and developed in groups. It would be interesting, therefore, to examine how narrative 
DST works for students who select the course for their major or minor studies and get involved in the project on a voluntary basis.

As this is an initial study, the overall goal is to explore DST integration by raising questions and making hypotheses concerning content, group work and narrative development. Thus, rather than giving definitive answers, the study aims to set the ground in terms of methods and frameworks of analysis to be further tested and enriched in future interventions.

\section{THEORETICAL BACKGROUND}

Digital storytelling (DST) is a 21st century metaphorical adjectivenoun phrase that signifies mapping of two domain areas in the meaning making process. In this mapping, 'digital' signals the comparison between the domain of technology and that of telling stories [28, 29]. Here, storytelling involves multiple modes of expression through language and other symbols and media. Contrary to traditional visual and cinematic storytelling, digital technologies nowadays offer the possibility for interactive ways of telling stories online through the use of web-based platforms and internet services (e.g., [16]). In terms of language and communication, this means that sometimes the adjective of the metaphorical phrase is more marked (i.e., more important, thus, capitalized here, DIGITAL), when the storytellers are more interested in the technologies used. Other times, storytellers pay more attention to the development of the narrative, the story line and the characters. In these cases, the noun is more marked (i.e., STORYTELLING).

Group work. Group work has been extensively researched in CSEd with encouraging results concerning students' motivation, creativity, critical thinking etc. $[3,31]$ Based on previous research experience in the field, in this study we look into group work as an inherent element of narrative DST. Narrative DST is a process of multiple story lines. It is actually about stories within stories and, therefore, in group work students synthesize the stories of their own development. When students take up a project voluntarily, they do so to earn credits to contribute to the overall grading of the course. The starting point therefore is mainly utilitarian. Group work however is more than the sum of required outputs per group. More importantly, it is about developing a sense of belonging, or being part of a whole (i.e., the group, the class, the university). Group work therefore is not only about content knowledge production; it is about identification as well. Based on insights of group theorists in social psychology and psychoanalysis (e.g. [5, 14, 17]), high levels of identification can increase leadership in the group. This, in turn, generates more possibilities for innovative/creative thinking.

Based on these, another research interest of the study is to investigate how group work relates to the development of the narrative and the representation of the central concept in the students' digital stories. For this purpose, we will define levels of identification on the basis of time spent on common tasks and in face-to-face meetings and the types of leadership developed in the groups. To do so, we will draw from data resulting from student manuscripts, interviews and digital stories.

Topic: recursion. In this study, we depart to examine how the elaboration of the plot (i.e., story line) and character development relate to the representation of the central concept (i.e., recursion),

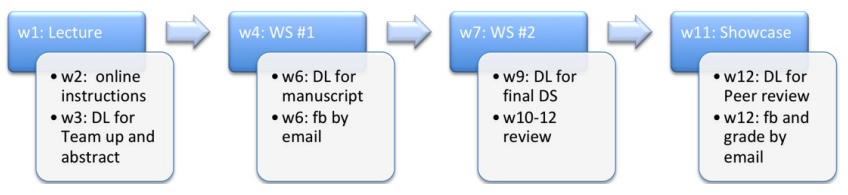

Figure 1: The first lecture in week 1 (w1) introduced the project briefly. Workshops (WS) introduced the project and DST in a more detailed level. Deadlines (DL) and feedback (fb) were planned according to the face-to-face meetings.

when the students work in DST groups. In other words, we look into whether and to what degree deliverables such as abstracts, manuscripts and final stories result in a balanced version of the metaphor, where both parts of the phrase are marked (i.e., DIGITAL STORYTELLING). Our selection of recursion as the central theme of DST was based on the fact that the concept is fundamental for the Data Structures and Algorithms course. Although other themes are possible, many notions are related to or are dependent on recursion. In addition, our previous experience of integrating DST into the course ([29]) showed that it was challenging for students to deal with both selecting from a long list of topics and the complexity of the DST process itself. Narrowing down the options reduced the overall complexity. Moreover, assigning only one topic served the research purposes. In this way, it was possible to compare students' digital stories in terms of character and narrative development.

\section{AIMS AND METHODS}

This study aims to discuss and analyze the implementation of a digital storytelling (DST) project within a narrative framework and group work. To do so, we will focus on the organization of student group purposes and patterns of work, and the development of plot and character in video stories aiming to explain recursion to peers and other audiences. The study aims to seek responses to the following research questions:

(1) In what ways do students develop the plot and the main character(s) in their digital stories?

(2) What role does student group work play in the development of digital stories?

(3) In what ways does student group work and narrative development relate with representations of recursion?

Course context and participants. This study draws from the Data Structures and Algorithms course targeting 2nd year CS minor students in Aalto University, Finland. The prerequisite for this course is at least one programming course (typically taught in Python). Many students enroll in this course even though it is not compulsory in their study program. However, not all registered students complete the course. For the Fall term 2018, the total enrollment was 289 students. About half of the students (140) passed the course. The low percentage is due to the fact that the course is not compulsory for most of the students.

Figure 1 shows the course time line and events. During the 12 week semester, the course had 6 lectures, 2 workshops, and weekly lab sessions (students divided to 6 lab groups). The focus of one of the 6 lectures was on the basics of DST, building the narrative structure and developing the story-line/plot and the characters. 
The workshops aimed to give time to the groups to present their ideas and plans and receive feedback from peers and the instructors for revisions and improvements. During the first workshop, the groups presented draft ideas, while the plans were more concrete in the second workshop. In addition, group formations became more stable in the second workshop. As some group members withdrew their participation in the project, members who decided to stay had to be integrated into other groups to meet the threshold for participation (i.e., 3-5 students/group).

For other activities than group work, the course had lab sessions. The lab sessions were targeted to students seeking help for the weekly assignments, most of which were automatically graded. The course has a long history of using automatically graded assignments, which also has changed the student behavior during the years. Many students take the course almost completely on-line, which makes unnecessary to travel to campus or meet other students. Especially, automatic assessment $[1,13]$ is very well received by the students, and it has shaped the course participation a lot.

In addition to automatically graded assignments, the course had a range of voluntary extra exercises, assignments, and a project for those aiming to best grades (i.e., above 3/5). Although, almost all the assignments were supposed to be individual work, DST was utilized to produce a framework for the project done in small groups. However, it was not the only voluntary exercise, and students needed to consider which options to take in case they wanted to pursue the best grade 5/5. Thus, we assume the project attracted especially those students more willing to participate in team work compared with those who might prefer individual work.

During the course period (October-December 2018), 64 unique students $(22 \%)$ participated in closed labs at least once. In addition, as blended learning methods were applied ([4]), the students had the option to ask for guidance in an on-line discussion forum integrated into the learning management system. 184 students enrolled into the discussion forum during the course $(64 \%)$. Thus, the preferred mode to study and ask for support for individual work was on-line.

Demographics. The students were mainly minor students from the School of Business (BIZ), School of Chemical Engineering (CHE), School of Electrical Engineering (ELE), School of Engineering (ENG), and School of Science (SCI). Twelve groups (44 out of 289 students, which is $15 \%$ of the students) started the DST project and submitted an abstract (First Draft). Each of the 12 groups consisted of 3-5 students. 8 out of 12 groups submitted a manuscript that included a draft plan of DST tasks. 7 groups out of 8 completed their project by submitting a video. These 7 groups also submitted an improved version of their text-based manuscript along with the digital story. In addition, each student reviewed other groups' manuscript and digital story after submitting their own.

Our study focuses on the 28 students in these 7 groups (appr. $10 \%$ of the whole course). The overall male-female ratio was not significantly different in project groups compared to the whole course (about $79 \%$ compared with $21 \%$ in both). All groups had male members, and 4 groups had 1-2 female members. The average age was 25 years. The ages varied between 20 and 39 years. The two oldest students were in the same group. In the rest of the groups, the ages varied between 20 and 27 years. The average age is quite high. This is natural as the course is typically took in the second year, and many students drop it and try again. 5 out of the 28 students that took the project were in this course the second or third time.

16 out of 44 did not complete the project, which is $36 \%$. This is rather high percentage, but it is explained by the fact that the project was not a compulsory part of the course. In addition, the course included other tasks that could be taken in order to achieve the desirable grade. Students had the chance to compare work load in different tasks and make the final decision to take the project or not. Some students felt that the work load for the project was too high compared to other alternatives.

The estimated work load was based on European Credit Transfer and Accumulation System (ECTS). The course was 5 credit point course, and one credit point-that is approximately 25-30 hours of work-was allocated to the project. Thus, a group having 4 members should have invested all together some 100-120 hours to the project. This was monitored in each phase as the groups were expected to report time-on-task in each submission. In the team up phase, they needed to plan their timings in the abstract. In the manuscript and the final submission, they needed to report on the time-on-task for each member.

Data analysis. The study aims to offer an analysis of content of data resulting from students' interviews, manuscripts and digital stories. There were 7 manuscripts (i.e., abstracts and reports) and 7 digital stories in video format, resulting from the work of 7 groups. Out of these, 4 groups and 11 students participated in 4 interviews. Each interview lasted approximately 40-45 minutes. For research purposes, the interviews were transcribed and anonymized. The transcriptions were sent to the participants to check and comment. The participants made it explicit under what copyright and licensing they shared their digital/video stories on the Web, with teachers and peers, and other audiences.

For the analysis, we moved inductively from more concrete to more abstract codes, themes and categories [7, 25]. With regard to the frameworks of analysis, we used thematic analysis to code and categorize interview data. Following transcription, excerpts from the interview data set were highlighted and coded manually. Then the coded excerpts were transferred to an Excel worksheet and set into larger categories and themes. A similar process was used for manuscript data. Then categories from interview and manuscript data were compared. In addition, discourse analysis was applied to the manuscripts in order to get a better insight into attributes of categories. When, for instance, the focus was on character development, we looked into the structure of student written expression in the manuscripts. Some manuscripts hardly mentioned the characters. Some others used complex main sentences and subordinate clauses with action verbs to elaborate on character development. To cross-examine findings we moved abductively back and forth from manuscripts to video stories to student interviews.

For reliability, both authors/researchers went through all the data sets. One researcher coded for group work and narrative development, then discussed emergent themes and categories with the other researcher. The same process was repeated for the analysis of representations of recursion where the other researcher took the lead. It was a joint decision what data to include and what to exclude from this paper. Although, the process was performed inductively, the description and discussion of results follows a deductive logic. 


\section{FINDINGS}

In the following Subsections 4.1-4.3, we describe our observations from three different point of views. These correspond to the three research questions we have. The results are combined in Table 1.

\subsection{The narrative in digital storytelling}

Types of digital stories. The analysis shows that group work had different orientations in planning and decision making about character development and story elaboration, and resulted in three types of digital/video stories. (1) Short films (G4 and G7). Two groups describe their story as short film aiming to educate and inspire audiences by combining unfamiliar (i.e., recursion) with familiar (e.g., Koch snowflake) elements into brief and fun videos. In addition to educating audiences, the short films aim to increase group's own awareness and knowledge of the topic through the articulation of definitions, properties and features in easily understandable language and format. To this end, the students use real-world examples in such ways that no prior knowledge of the concepts is required. (2) Documentary-style stories (G1 and G2). Two groups came up with videos that give emphasis rather on a 'fact-based' representation of recursion than a character-plot interaction. The narrative is linear and built on alternating still images. The storytelling is done through voice over (G1), and with a student-actor who provides the account of events (G2). (3) Program-based stories (G3, G5 and G6). The narrative here is based on selection, which means that it is built on if-then-else (i.e., conditional) basis. When a character meets an issue, for instance, possible solutions are not presented or elaborated in detail. Instead, the narrative moves forward into the next character or the next event based on a programmed condition. Typically the submissions of this type of story was not a manuscript (PDF) including a link to the video, but a zip file including also the software to execute the story (e.g., an interactive game).

Character development. For character development, we looked into language use in student manuscripts. For instance, G7 use action verbs and complex grammatical structures and adverbials to describe how characters develop: The story deals with Essi, who is a student who always leaves preparing for an exam to the very last minute... Having heard of recursion she panics and leaves her room to find assistance... In a similar way, G4 develop their characters through action verbs and elaborate structure. The narrative in these stories indicates a change in the characters' thinking and action and, therefore, the development is visible and clear. However, other groups' (e.g., G2) characters remain floating between a presenter's and a narrator's role. In this story, the character's role to plot development is not clear, although there is alternation from one event to the next. However, the story is a linear account of facts rather than a reconfiguration of the storytellers' ideas.

In stories where characters are not well-developed, an account of actions is missing or the need for a character is not explicit, e.g., in G3 story. This might relate to the fact that G3 work was more independent and based on a clear task division. This organization, although based on a well-articulated rationale, came up with unclear character whose relation to the events of the story was loose. Following a similar pattern, G5 work results in a mythical character whose purposes and actions remain undefined. Contrary to the way the narrative develops in G3 story, which is also based on the logic of conditional branching, the plot in the former is rather linear and closed-ended. Overall, in conditional-based stories, the main character is male and, instead of developing a 'life of his own', revolves around a right/wrong decision before moving on to the next choice.

The plot. To understand how plot was elaborated, we looked into event descriptions in the manuscripts. The plots in short films were well-articulated with complex structure. Also, the programbased story of G3 uses complex sentences and dependent clauses. However, despite the elaborate expression, the background of this story is made available only in the manuscript, which might lead the audience to a partial understanding of what is going on, unless the written text is available. For G3, getting the concepts straight meant the process would be smooth enough not to not have to go back and start all over again. This plot elaboration seems to have happened on a random basis at the expense of concept representation. The students in G7 expressed similar views in the interview about the narrative-concept relation. Despite this overlap, their short story balanced character development and plot elaboration, and resulted in clear representations of central concepts.

Another indication of a well-elaborated plot is the element of surprise. Some groups recognized the need to include elements of surprise through accounts of unexpected events leading to different configurations of the story. Adversities, however, remain underdeveloped in the G5 programmed story and mainly concern the obstacles the character falls over when running the maze. Groups 4 and 7 also use adversities, only in a more elaborate way. For instance, G4 write, However, Admin ends up in the trouble as there is no safely way to get back down. Admin's travel partner Network Specialist offers to help...

\subsection{Group work in narrative digital storytelling}

Joint planning and decisions involved dividing the work into more concrete tasks. As G1 write in their manuscripts: As a group, we quickly decided that the work packages for the project involved voice acting, script writing, research, video editing and organizational tasks. As a group it was determined that this division of work packages covered everything related to the project. Splitting the tasks led to different tactics among the groups similar to those described by Waite et al. [31] as: Sequential segmentation (SS, "I work on it for a while, then pass it along to you."); Parallel segmentation (PS, "We break it up and everyone does a piece."); Natural selection (NS, "We each carry it out and then choose the best result, or we choose the best person and let them do it."); and, Collaboration (Co, "We interact closely during the task.").

As Waite et al. [31] put it: "In each of the first three cases, the members of the group are effectively working alone. Working alone is our students' preference [...] and therefore whenever they are given a task they actively employ one or more of those three tactics regardless of the intent of the professor." However, we can see real collaboration as well, for example, in G7. As the members of this group said in their interviews: I-ee 3: I've done quite a few groupworks during my studies and this one was different... In these you just divide the tasks, everyone does their own part and then you just put things together. In that sense, this was a nice change... 
Table 1: Narrative development, group work (GW) and representations of recursion.

\begin{tabular}{|l|l|l|l|l|l|l|}
\hline Type of story & Character & Plot & Tactics & GW & Recursion in video & R. in manuscript \\
\hline Short film (G4, G7) & well-developed & well-elaborated & Co & CP, MR & clear & clear \\
Documentary (G2) & not clear & linear & Co & CP, MR & clear & not defined \\
Documentary (G1) & not clear & linear & PS & CF & explicit misconception & not defined \\
Program (G5) & not well-developed & well-elaborated & Co, NS & CP, Am & no misconceptions & no misconceptions \\
Program (G6) & not well-developed & well-elaborated & Co, NS & CP, Am & no misconceptions & not defined \\
Program (G3) & not well-developed & random & PS, NS & CP, FL & no misconceptions & no misconceptions \\
\hline
\end{tabular}

Overall, the way tasks were allocated and the time students spent together on face to face (f2f) group work resulted in different types of group leadership and identification, i.e., an individual's sense of belonging to the group. See Table 1 . We denote the leadership centripetal $(\mathrm{CP})$ in case we see distributed type of leadership in collaboration, or centrifugal leadership (CF) that typically occur in case the tactics is sequential segmentation (SS), parallel segmentation (PS), or natural selection (NS). For identification, we have analyzed the group work in order to observe mutual recognition (MR) among group members.

Group work and leadership. Most groups (G4-G7) both shared and divided into distinct tasks. Two groups (G2 \& G3) had only shared tasks, while G1 split the work into completely distinct tasks, which they coordinated through different Web platforms and software (e.g., telegram, google docs, WhatsApp). This allocation led to a centrifugal leadership (CF), in the sense that, although the overall purpose was met (i.e., the digital story was completed and submitted), evidence for a common purpose is scant. The fact that the members of G1 hardly met f2f kept identification at low level.

On the other hand, the groups that shared or both shared and divided tasks, developed a centripetal (CP) type of leadership. This means that leadership was distributed among the group members. More particularly, in addition to attending lectures and workshops, G2 and G7 spent more than 2 and 4 hours in face-to-face (F2f) meetings, respectively. F2f time allows to grow this something in common that makes group identification between group members possible [14]. Spending time together, therefore, seems to have led to more concrete sets of common purposes. As a result, mutual recognition (MR) among group members has also grown. Mutual recognition became evident in groups 2, 3 and 7 interviews, where the members acknowledged one another's work and contributions. This means that the degree of identification is higher in these cases.

Also, in the case of G3, where the group looked up to the peer with more working hours on programming, leadership is more focused (FL) than distributed. It seems that a kind of transference has occurred in this case, in the sense that the students recognize a certain binding quality in their peer. Although this is a different enactment from the case of G7, where all the members are poles of leadership, both G3 and G7 are evidences of student interest in - at least- as much in the object of study as in the grading points. It is therefore the object of study (i.e., programming) that constitutes an element of unification as well. Finally, in the cases where time spent together remains unconfirmed (i.e., in interviews or manuscripts, G5 and G6), mutual recognition is ambiguous (Am), despite the fact that group leadership seems to be centripetal.

\subsection{Representations of recursion and code}

None of the digital stories covered or visualized stack frames or some other representation for how to implement recursion in computing. This was partially due to the assignment: we explicitly asked examples of recursion in nature. Thus, the projects show more external examples of recursion than internal implementation details even though many projects included also source code. Thus, the digital projects focused on showing what the code does rather than how it does it. Another reason for this might be in the fact that the students were minor CS students. Major students might have different approach to technical representations.

One of the challenges in representing recursion is that its outer behavior does not differ from iteration, i.e. for- and while-loops in algorithms. This seems to be very hard to grasp for the students, but even harder to express in their digital stories. In addition, some of the stories included explicit function calls, but it was unclear if they were recursive or just a function calling another function, calling another function, etc. This shows up in some projects which hold explicit misconceptions about recursion even though they got feedback during the project. For some reason, they did not pay attention to the critic received.

We also evaluated the manuscripts and videos according to the accuracy of the terminology as well as representation in Table 1. In some videos and manuscripts we can see (explicit) misconceptions such as missing base case (or cases) or the recursive steps do not reach the base case(s). Some representations had possible misconceptions related to the fact that neither the manuscript nor the video made it clear whether the story actually represents recursive or iterative process. This is kind of implicit misconceptions as the task was to represent recursion. The groups did not make adequate distinction between these two. The third category includes the rest of the stories that were considered correct. They cannot be misinterpreted to be iterative processes even though there is no actual recursive algorithm represented in the manuscript. However, most of this type of stories included also recursive code fragments to illustrate recursion.

\section{DISCUSSION AND CONCLUSIONS}

Table 1 combines the results and it is sorted according to the type of story. The groups that produced short films developed a centripetal (CP), distributed type of leadership, while mutual recognition (MR) among group members was high. More particularly, in relation to types of stories, digital stories that were categorized as short films displayed well-elaborated characters and well-developed plots. In addition, the representations of recursion were very clear both in 
Table 2: Types of DST. There seems to be correlation among emphasis of DST, types and accuracy of digital stories.

\begin{tabular}{|c|c|c|c|c|}
\hline Emphasis & Type of story & Identification & Narrative & Representation/recursion \\
\hline $\begin{array}{l}\text { DIGITAL STORYTELLING } \\
\text { digital STORYTELLING } \\
\text { DIGITAL storytelling }\end{array}$ & $\begin{array}{l}\text { Short film } \\
\text { Documentary } \\
\text { Documentary } \\
\text { Programmed }\end{array}$ & $\begin{array}{l}\text { high } \\
\text { high } \\
\text { low } \\
\text { high/NS }\end{array}$ & $\begin{array}{l}\text { well-elaborated } \\
\text { fact-based but linear and unclear } \\
\text { fact-based but linear and unclear } \\
\text { well-thought plot but loose characters }\end{array}$ & $\begin{array}{l}\text { well-represented } \\
\text { no explicit misconception } \\
\text { explicit misconception } \\
\text { possible misconceptions }\end{array}$ \\
\hline
\end{tabular}

the manuscript and video. In documentary-like stories, the plot was fact-based and linear with ambiguous characters whose development remained unclear. The groups that produced this story genre displayed two types of leadership. When the leadership was moving outside the group and was, therefore, centrifugal $(\mathrm{CF})$, the level of identification was low. This group work resulted in an explicit misconception of recursion. On the contrary, the documentarylike story of the group with tighter coordination of activities and face-to-face meetings displayed no explicit misconception. It seems, therefore, that face-to-face collaboration influences the group narrative, which, in turn, influences the grasp of the central concept.

Group work and narrative development in programmed stories overall confirm this finding. It seems, however, that the stories were in the middle of the way. Although the characters were better defined than in documentaries, they were not as well-developed as in short films. Although, the plots were well-elaborated, they were conditional with binary alterations of a 'yes/no' type. Moreover, the type of leadership seems to have been centripetal. There was scant evidence concerning time spent together or how common tasks were dealt with or if common tasks existed at all. Thus, mutual recognition remains questionable for two out of the three groups of this category. Contrary to this, the third group's interview provided evidence of mutual recognition. Moreover, this group is an example of Natural Selection type of tactics. However, this group work resulted misconceptions in the description of recursion in the manuscript. A possible explanation could be that misconception went unnoticed, as, along with leadership, the 'responsibility' for successful task completion was transferred to a single peer/leader.

Overall, the students who were interviewed described the multilayered task of representing recursion by using digital technologies and telling stories as a positive learning experience. During interviews, the participating students expressed the belief that they had grasped a better understanding of recursion through the process of documenting ideas in narrative form (i.e., abstracts, manuscripts and video stories). Developing the story line seemed to be more important than characters at first; however, three groups mentioned that the role of the character became clearer to them during the second workshop. Then they made changes by further elaborating the stories. As this was an initial study, measurement of the learning outcomes mainly took place through the interviews. Nevertheless, as we see the digital stories themselves as documentation of learning, we will use insights from the representations of recursion to build our future endeavors. Besides, this was one first step to better understand differences between automated assessment and qualitative evaluation (e.g., through peer feedback). Future interventions, then, could include control and experimental groups to compare the two types of evaluation of learning a common concept. Considering the above, three versions of DST have resulted in relation to types of stories, group narratives and representations of recursion (See Table 2) :

DIGITAL STORYTELLING. Group work that displays recursion in short films relates to a high level of identification and a narrative of mutual recognition. Leadership is distributed among group members and the development of characters and plot are of equal importance. In this approach, the representation of recursion is both clear and accurate.

digital STORYTELLING. These emerge out of documentary-style stories, with a fact-based representation of recursion, alternating still images and an elaborated, but linear, plot. In this case, the use of digital technologies was narrow in the production and/or the editing process. Technology overall seems to have played a minor role, as there was no code in the groups' manuscripts. This result correlates with two distinct types of leadership. Centrifugal leadership keeps the level of group identification low and comes up with a misconception in the representation of recursion. Centripetal leadership leads to an accurate representation of recursion.

DIGITAL storytelling. This version emerges out of programmed stories, where a lot of attention was given on programming and code development. In these stories, the use of technology played an important role. However, character development was unclear and loose, although the plot well-elaborated. In addition, identification with the group leader and natural selection of leadership seems to relate with misconceptions in the way recursion was represented in the video story.

\subsection{Validity issues}

The project was not the only voluntary exercise to achieve the best grade $5 / 5$, thus many students considered carefully which options to use. As a voluntary exercise, this brings certain bias to the population. This is evident if we look at the final results. 23 out of 28 students $(82 \%)$ have passed the course at the time of writing. The rest 5 have not taken the final exam or re-exam, which is required to pass the course. Thus, the percentage is going to be even higher after the last re-exam in September. This is significantly different from the overall passing rate that is around $50 \%$. Thus, we can conclude that those students taking part in the project were not only more motivated to achieve a good grade, but also more motivated to pass the course compared with students that did not choose the project. Moreover, due to the low number of groups, our findings are more hypotheses than definitive results. It would be good to see replication studies in the future to confirm or falsify our findings. This would help us to figure out how to introduce this kind of project work in HE including continuous orientation and motivation, support for group work (e.g., workshops), encouragement to attend campus events, etc. At least we can say that DST is a promising approach to allure some students back to campus. 


\section{REFERENCES}

[1] T. Auvinen and A. Korhonen. 2014. Rubyric Assessment and Annotation Tool. In EdMedia: World Conference on Educational Media and Technology. Association for the Advancement of Computing in Education (AACE), Waynesville, NC, 1087-1091.

[2] P. Dourish. 2016. Algorithms and their others: Algorithmic culture in context. In Big Data \& Society. SAGE Publications, open access, 1-11. https://doi.org/10. $1177 / 2053951716665128$

[3] H. Drury, J. Kay, and W. Losberg. 2003. Student satisfaction with groupwork in undergraduate computer science: do things get better?. In Australasian Computing Education Conference (ACE2003), T. Greening and R. Lister (Eds.). Conferences in Research and Practice in Information Technology, Adelaide, Australia, 9 pages.

[4] C. Dziuban, C. Graham, P. Moskal, A. Norberg, and N. Sicilia. 2018. Blended learning: the new normal and emerging technologies. International fournal of Educational Technology in Higher Education 15, 3 (2018). https://doi.org/10.1186/ s41239--017--0087--5

[5] S. Freud. 2001. Group Psychology and the Analysis of the Ego (1921). In The Standard Edition of the Psychological Works of Sigmund Freud, J. Strachey (Ed.). Vol. XVIII. Vintage, London, UK

[6] T. Gillespie. 2016. Algorithms, clickworkers, and the befuddled fury around Facebook Trends. https://socialmediacollective.org/2016/05/18/facebook--trends/.

[7] B. G. Glaser and A. L. Strauss. 1995. The Discovery of Grounded Theory: Strategies for Qualitative Research. Aldine Publishing Co, NY, USA.

[8] G. Hull and M. Katz. 2006. Crafting an agentive self: case studies of digital storytelling. Research in the Teaching of English 41, 1 (2006), 43-81.

[9] G. Jamissen, P. Hardy, Y. Nordkvelle, and Pleasants H. (Eds.). 2017. Digital Storytelling in Higher Education - International Perspectives. Springer International Publishing AG, Switzerland.

[10] H. Jenkins, M. Ito, and D. Boyd. 2016. Participatory Culture in a Networked Era, A Conversation on Youth, Learning, Commerce \& Politics. SAGE, Cambridge, UK.

[11] D. M. Kaplan. 2003. Ricoeur's Critical Theory. SUNY, N.Y., USA.

[12] R. Kitchin. 2017. Thinking critically about and researching algorithms. Information, Communication \& Society 20, 1 (2017), 14-29. http://dx.doi.org/10.1080/ 1369118X.2016.1154087

[13] A. Korhonen and L. Malmi. 2000. Algorithm simulation with automatic assessment. ACM SIGCSE Bulletin 32, 3 (2000), 160-163. DOI10.1145/353519.343157

[14] E. Laclau. 2005. On Populist Reason. Verso, London, UK.

[15] J. Lambert. 2013. Digital storytelling: Capturing lives, creating community. Routledge, NY, USA.

[16] A. Marwick and D. Boyd. 2014. Networked privacy: how teenagers negotiate context in social media. New Media \& Society 16, 7 (2014), 1051-1067.
[17] W. McDougall. 1920. The Group Mind. Cambridge University Press, Cambridge, UK.

[18] P. McGee. 2015. The instructional value of digital storytelling: Higher education, professional, and adult learning settings. Routledge, NY, USA.

[19] H. Niemi, V. Harju, M. Vivitsou, K. Viitanen, J. Multisilta, and A. Kuokkanen. 2014. Digital storytelling for 21st-century skills in virtual learning environments. Creative Education 5, 9 (2014), 657-671.

[20] J. Nuutinen. 2009. Nucleus Model for Designing Social Mindtools: Woven Stories. Ph.D. Dissertation. Faculty of Science, University of Joensuu, Joensuu, Finland.

[21] J. Penttila, V. Kallunki, H. M. Niemi, and J. Multisilta. 2016. A Structured Inquiry into a Digital Story: Primary School Students Report the Making of a Superball. International fournal of Mobile and Blended Learning 8, 3 (2016), 19-34.

[22] P. Ricoeur. 1992. Oneself as Another. University of Chicago Press, Chicago, USA.

[23] B. Robin. 2008. Digital storytelling: A powerful technology tool for the 21s century classroom. Theory into practice 47 (2008), 220-228.

[24] A. Sadik. 2008. Digital storytelling: A meaningful technology-integrated approach for engaged student learning. Educational Technology Research \& Development, 56 (2008), 487-506.

[25] J. Saldana. 2009. The Coding Manual for Qualitative Researchers. SAGE, London, UK.

[26] Z. Tufekci. 2016. Technology in Global Activism, Uprisings \& Social Movements. https://shorensteincenter.org/zeynep-tufekci/.

[27] M. Vivitsou. 2019. Constructing Identities in Online Encounters - A Study on Finnish \& Greek Young Students' Digital Storytelling Practices. International Journal of Teacher Education and Professional Development (IFTEPD) 2 (2019).

[28] M. Vivitsou. 2019. Digital storytelling in teaching \& research. In Encyclopaedia of Education \& Information Technologies, A. Tatnall \& J. Multisilta (Ed.). Springer, Springer Nature Switzerland AG.

[29] M. Vivitsou and A. Korhonen. 2018. Digital Storytelling for Socio-Technical Literacies: Participatory Methods in a Computer Science Higher Education Course. https://ssrn.com/abstract=3175925.

[30] M. Vivitsou, H. Niemi, G. Wei, V. Kallunki, and R. Miao. 2017. Teachers' practices to support student work in digital storytelling: A study on Finnish and Chinese school teachers' experiences. Seminar.net International fournal of Media, technology and Lifelong Learning 13, 2 (2017), 14-29. https://journals.hioa.no/index. $\mathrm{php} /$ seminar/article/view/2306

[31] W. M. Waite, M. H. Jackson, A. Diwan, and P. M. Leonardi. 2004. Student Culture vs Group Work in Computer Science. SIGCSE Bull. 36, 1 (March 2004), 12-16. https://doi.org/10.1145/1028174.971308

[32] J. Woodhouse. 2008. Storytelling: A telling approach in healthcare education. In The Narrative Practitioner international conference. open access, Wrexham, England, UK, 1-6. https://www.researchgate.net/publication/30067784_ Story-telling_a_telling_approach_in_healthcare_education 